\title{
El cultivo de soya y su importancia para el Ecuador
}

\section{The cultivation of soy and its importance for Ecuador}

\author{
Nelly Sayenka Vergara Diaz, \\ Universidad de Guayaquil, Ecuador \\ Fernando Rodolfo Orellana Intriago \\ Victor Hugo Vizueta Tomalá, \\ Daniel Antonio Mata Lopez, \\ David Alberto Bernal Paredes, \\ Pablo Ricardo San Andres Reyes \\ Consultor Independiente, Ecuador
}

Autor para correspondencia: nellysvergarad@gmail.com; psanandres@ecotec.edu.ec

Fecha de recepción: 12 de Septiembre de 2016 - Fecha de aceptación: 01 de Noviembre de 2016

Resumen: El objetivo del estudio fue observar la producción de soya en el Ecuador y sus oportunidades de exportación al mundo, la metodología fue la revisión bibliográfica de artículos referentes a la producción y comercialización, con datos estadísticos del mercado global, regional y local, se realizó una criba de selección de artículos de inclusión, determinando que estos no podían superar los cinco años de antigüedad, de estar publicados en revistas indexadas, pertenecer a la línea de economía y producción, los resultados hallados fue que la producción en el mundo fue a julio de 2016 de 325.95 millones de toneladas métricas por hectárea (tm/ha), siendo su mayor productor Estados Unidos con 105,596,000tm/ha, seguido de Brasil, con 103,000,000, Ecuador llegó a un porcentaje de $2.04 \mathrm{de}$ tm/ha, en comparación con EEUU y en relación a la producción de Brasil, el mayor productor regional (América del Sur). Esto permitió inferir que en el país no se ha tomado en cuenta la demanda a nivel mundial de esta oleaginosa, la Soya se constituye en una oportunidad de mercado para los productores y comercializadores de soya en el Ecuador, con lo cual se lograría participar en el plan nacional de buen vivir del ecuador Plan Nacional del Buen Vivir y a contribuir a la sublínea de investigación de economía y emprendimiento social, sostenibilidad y gestión territorial. Palabras claves: producción; comercialización; soya; exportación

\begin{abstract}
The aim of the study was to observe soybean production in Ecuador and export opportunities to the world, the methodology was a literature review of articles about the production and commercialization, with statistics data of global, regional and local market. Some articles for inclusion were selected, determining that these could not exceed five years old, to be published in indexed journals, belong to the line of economy and production, the found results showed that production in the world was to July 2016 of 325.95 million metric tons per hectare $(\mathrm{mt} / \mathrm{ha})$, and its main US producer $105,596,000 \mathrm{tm} / \mathrm{ha}$, followed by Brazil with 103,000,000, Ecuador reached a rate of $2.04 \mathrm{mt} / \mathrm{ha}$, compared to the US and in relation to the production of Brazil, the largest regional producer (South America). This allowed to infer that the country has not been taken into account the demand worldwide of this oleaginous. The soybean constitutes a market opportunity for producers and marketers, which could be achieved to participate in the national plan of good living in Ecuador and contribute to the sub-line research of economy, social entrepreneurship, sustainability and territorial management.
\end{abstract}

Key words: production; commercialization; soybean and exports 


\section{Introducción}

La soya es una leguminosa de origen asiático, su composición es de alto contenido proteínico y graso medio, consumida como fuente de nutrientes en la alimentación humana y en las últimas décadas se ha venido empleando como importante insumo para producir alimento para el ganado en mayor escala de avícola y porcino. En la nutrición humana se consume en su forma básica de gramínea o en procesados tales como aditivos de sabor, carne de soya, se extrae aceites por sus contenidos grasos es muy recomendado. A partir de la soya existen industrias que la cuentan como sus insumos para la producción de dulces, confitería, repostería, galletas. También existe actividad en la industria de producción de harina de soya y sus derivados.

Según las cifras publicadas por la FAO (FAO, 2015), en términos de superficie cosechada y volumen producido los principales productores de soja en grano a nivel mundial en el año 2014 fueron Estados Unidos, Brasil, Argentina, China e India (en ese orden). Sin embargo, los países con mayor productividad en el cultivo en ese mismo año fueron: Tailandia, Turquía, Italia, Serbia y España con rendimientos entre 3.4 y 6.3 toneladas por hectárea.

La soya es una oleaginosa de demanda mundial cuyo potencial no está siendo considerado en Ecuador. Ecuador posee condiciones excelentes para su producción según el informe de Rendimientos Y Características De Soya En El Ecuador Verano (Ministerio de Agricultura, 2015): "El rendimiento a nivel nacional para el ciclo de verano del 2015 se determinó en $2.04 \mathrm{tm} / \mathrm{ha}$. La provincia de mayor rendimiento fue Los Ríos con una producción de 2.16 toneladas por hectárea. Los cantones que se destacaron con un rendimiento superior a la media nacional fueron Baba y Vinces en Los Ríos y Urbina Jado en Guayas. En contraste, los cantones de menor rendimiento fueron Ventanas y Pueblo Viejo en Los Ríos y Milagro en Guayas. Las características de los productores de soja ecuatorianos en el ciclo de verano del 2015 fueron: siembra bajo sistema convencional sin nivelación o acceso a riego de una superficie promedio de 6.41 hectáreas; utilización de semilla en su mayoría correspondiente a la variedad P34 e implementación del método de siembra al voleo; fertilización del cultivo con la aplicación bastante homogénea de fertilizantes nitrogenados, fosforados y potásicos; y mecanización de la preparación del suelo. El principal problema reportado por los agricultores fue la Roya."

El presente de trabajo de investigación se realizó en base a una investigación documental bibliográfica, recopilada de fuentes internacionales y del Ecuador.

\section{Métodos}

Para esta investigación se utilizará el método deductivo que nos permitirá interpretar y comprender las particularidades del tema planteado.

Se utilizarán la investigación documental y descriptiva considerando que tenemos como objeto de estudio la producción de soya en el Ecuador y sus oportunidades en el mercado mundial. La Investigación descriptiva "Se reseñan la características o rasgos de la situación o fenómeno objeto del estudio" (Bernal, Cesar A, 2010) y la Investigación Documental "La investigación documental consiste en un análisis de la información escrita sobre un determinado tema, con el propósito de establecer relaciones, diferencias, etapas, posturas o estado actual del 
conocimiento respecto al tema objeto del estudio.” (Bernal, Cesar A, 2010). a través de la obtención de la información para la investigación por medio de la observación, internet y análisis de documental del tema planteado y a través de los datos obtenidos de la página de páginas web.

\section{Resultados}

\section{Tendencias de la Demanda}

\section{El incremento en la población mundial}

La teoría demográfica trata de explicar la dinámica del crecimiento de la población, por medio de postulados que explican el fenómeno de sus cambios y evolución, estructura y características desde el punto de vista cualitativo -sociológico y económico- como desde el cuantitativo o estadístico. A principios del siglo XVII Thomas Malthus (Malthus, 17661834)pronuncio que el aumento de la población es en progresión geométrica y el de los recursos para la subsistencia es en progresión aritmética, y de no tomarse acciones con el advenimiento de nuevas vidas humanas se haría cada vez más precaria la subsistencia hasta llegar a su extinción.

\section{Mejora en la calidad de vida}

La teoría de la transición demográfica, identifica con la revolución industrial una afectación evidente en la merma de mortalidad en la población humana y optimización de condiciones de vida por la tecnología, alimentos, mejora en sanidad, transportación, acceso a medicinas, etc., y el decrecimiento de la natalidad vinculado a los cambios sociales y económicos que han dado lugar a progresos en urbanización, industrialización, incremento de escolaridad, empleo, en particular del género femenino. En la evolución histórica de los últimos dos siglos, hasta hace unos 40 años en países subdesarrollados, estos cambios han traído consigo aparejadas alteraciones de comportamiento en la natalidad y mortalidad de ser elevadas, hasta la época actual en que ha descendido la natalidad y la mortalidad.

En su primera fase de la sociedad preindustrial la tasa de la natalidad y mortalidad es muy alta, por lo que el crecimiento poblacional es bajo y lento.

En la Segunda Fase inherente a países en vías de desarrollo, comienza la transición, irrumpen en el mundo las condiciones que darán paso al crecimiento de la población, gracias a las mejoras tecnológicas, incremento de los rendimientos en la producción de agroalimentos, acceso a medicina y educación. Mejora en la esperanza de vida al nacer.

Hallamos en la Tercera fase, la consumación de la transición, declive significativo de la natalidad, impulsado por la inclusión de la mujer en la educación, la fuerza laboral, anexo a otras mutaciones sociales.

Cuarta fase, régimen demócrata actual, ultimo estadio característico de la sociedad postindustrializada en la mortalidad se muestra más controlada y diríase que hasta manejable con los avances en tecnología y ciencia. 


\section{Sofisticación de la Alimentación}

Con el advenimiento del desarrollo de las sociedades y sus cambios sociales de diversidad de configuración en los núcleos familiares, hábitos de consumo y preferencias alimentarias, esto ha mutado, ha mega diversificado y multiplicado exponencialmente la demanda de consumo de agroalimentos. Pasando de la alimentación por supervivencia de la primera fase a la alimentación caracterizada por las necesidades de las sociedades modernas, propias de la cuarta fase.

Hoy existe la demanda de agroalimentos para fabricar productos alimenticios para:

Consumo animal

Biocombustibles

Comida para mascota

Consumo humano

\section{Explosión Demográfica}

De acuerdo al informe de perspectivas de la población mundial de la Organización de Naciones Unidas, (ONU, 2015) la cifra de habitantes en el mundo pasará de 7.349 estimada en el año 2015 a 8.501 millones de habitantes en el año 2030. Por regiones la de mayor expansión será África, seguida de Asia y tras esta Europa. El crecimiento demográfico crea mayor demanda en la producción de alimentos.

\section{Proyección De Población Mundial}

(Expresada en millones de habitantes)

Tabla 1. Elaborado por el autor

\begin{tabular}{lllll}
\hline AREAS & $\mathbf{2 0 1 5}$ & $\mathbf{2 0 3 0}$ & $\mathbf{2 0 5 0}$ & $\mathbf{2 1 0 0}$ \\
\hline World & 7349 & 8501 & 9725 & 11213 \\
África & 1186 & 1679 & 2478 & 4387 \\
Asia & 4393 & 4923 & 5267 & 4889 \\
Europe & 738 & 734 & 707 & 646 \\
América Latina y el Caribe & 634 & 721 & 784 & 721 \\
Norte América & 358 & 396 & 433 & 500 \\
Oceanía & 39 & 47 & 57 & 71 \\
\hline
\end{tabular}

Fuente: Perspectivas de la Población Mundial año 2015 Informe de la Organización de Naciones Unidas (ONU, 2015)

\section{Producción de Soya a nivel mundial}

La producción y comercialización de la Soya a nivel mundial, constituye una oportunidad de desarrollo económico para los países agrícolas, la proyección de producción mundial de soja (Soja Producción Mundial, 2016)

\section{Proyección De Producción Mundial De La Soya}

(Expresada en toneladas métricas) 
Tabla 2. Elaborado por el autor.

\begin{tabular}{lll}
\hline Puesto & País & tm/ha \\
\hline $\mathbf{1}$ & Estados Unidos & $\mathbf{1 0 5 , 5 9 6 , 0 0 0}$ \\
$\mathbf{2}$ & Brasil & $103,000,000$ \\
$\mathbf{3}$ & Argentina & $57,000,000$ \\
$\mathbf{4}$ & Otros & $21,204,000$ \\
$\mathbf{5}$ & China & $12,200,000$ \\
$\mathbf{6}$ & India & $11,700,000$ \\
$\mathbf{7}$ & Paraguay & $9,000,000$ \\
$\mathbf{8}$ & Canadá & $6,250,000$ \\
\hline \multicolumn{2}{c}{ Fuente: } & (Soja Producción Mundial, 2016)
\end{tabular}

El presente mes de septiembre de 2016 el Departamento de Agricultura de Estados Unidos (USDA) estimó que la Producción Mundial de Soja 2016/2017 será de 330.43 millones de toneladas métricas, cerca de 0.01 millones de toneladas más de lo estimado el mes pasado.

La Producción Mundial de Soja (*) del año pasado fue de 312.97 millones de toneladas. Los 330.43 millones de toneladas estimados este año podrían significar un incremento de 17.45 millones de toneladas o un 5.58\% en la producción de soja alrededor del mundo. (Soja Producción Mundial, 2016)

\section{Demanda de soya a nivel mundial}

La India es el país con mayor población vegetariana, un 40\%. Según un informe de la Fundación Foodways (Foodways, 2015), en Estados Unidos actualmente es el 13\% de la población. En Inglaterra, el $47 \%$ de la población se autodenomina vegetariana. El 28\% de brasileños "están procurando comer menos carne".

Hay más de 600.000 millones (y en aumento) de vegetarianos en el mundo. "En Alemania hay cerca de 6 millones de vegetarianos, aproximadamente un $8 \%$ de la población." Son palabras de Sebastian Zösch, presidente de la Vegetarier Bund Deutschland, la Unión Vegetariana Internacional. (Union Vegetariana Internacional, 2016) Dicha Unión ha calculado que el número de vegetarianos aumenta un $10 \%$ cada año.

Se nota, desde el punto de vista empresarial, en el aumento de productos alternativos a la carne, y también en la cantidad de socios de la Unión Vegetariana.

Esto demuestra que en los países de los distintos continentes existe la marcada tendencia hacia la concepción de un estilo de vida más saludable de respeto por la alimentación y de conciencia de convivencia sustentable con el medio ambiente.

\section{Proyección de soya a nivel mundial}

Con respecto a la carne de soya, aun no existe una oferta de este producto establecida a nivel mundial. Sin embargo, es preciso mencionar a los principales países exportadores de la soya para tener una idea más clara de la cantidad a nivel mundial de las exportaciones de la soya. 
En la siguiente gráfica se demuestra a los principales países exportadores de la soya y con su respectiva proyección de las exportaciones hasta el año 2023.

\section{Proyección De Principales Países Exportadores De La Soya}

(Expresado en millones de toneladas métricas)

Tabla 3. Elaborado por el autor.

\begin{tabular}{llll}
\hline Exportaciones & $\mathbf{2 0 1 3} / \mathbf{1 4}$ & $\mathbf{2 0 1 7 / 1 8}$ & $\mathbf{2 0 2 2 / 2 3}$ \\
\hline Brasil & 39,4 & 48,9 & 63,8 \\
EEUU & 41,2 & 42,9 & 43,8 \\
Argentina & 10,9 & 14,2 & 17,5 \\
Otros Sudamérica & 7,8 & 9,3 & 11,3 \\
Ucrania & 1,9 & 2,5 & 3,2 \\
Otros & 3,8 & 4,1 & 4,7 \\
Total Exportaciones & 105 & 121,9 & 144,3 \\
\hline \multicolumn{2}{c}{ Fuente: (Agrodigital, 2015) }
\end{tabular}

EEUU, Brasil y Argentina lideran la producción y el comercio mundial de la soja. En 2009 y 2010 las exportaciones de estos países representaron el 90\% de las exportaciones por un total de 82 millones de toneladas métricas. EEUU, ocupó en esta campaña un primer lugar con 38,1 millones de toneladas métricas de soja en grano exportadas, Brasil con 25,3 millones de toneladas métricas estaría en un segundo lugar y Argentina con 8 millones de toneladas métricas, en un tercer puesto en este ranking comercial.

\section{Cultivo de Soya en Ecuador}

El cultivo en Ecuador, de acuerdo a informe de Rendimientos Y Características De Soya En El Ecuador Verano (Ministerio de Agricultura, 2015) :

"El cultivo de soja en el Ecuador no se caracteriza por ser un cultivo tradicional, por lo que los productores reportan ser la primera generación en su familia que siembra este cultivo. De igual manera, el cultivo de soja en el Ecuador no se caracteriza por un alto nivel de especialización; por lo que 55\% de los productores de soja ecuatorianos complementan sus ingresos con otras fuentes de trabajo como la producción de otro cultivo, el comercio o empleo asalariado. De hecho, la mayoría de los productores realiza la siembra de soja una sola vez al año en época de verano como alternativa a la siembra de cultivos tradicionales como el arroz y maíz duro seco. Durante este ciclo de verano, el gasto promedio incurrido por los productores según la encuesta realizada fue de USD 790 por hectárea. El productor de soya ecuatoriano promedio se identifica por tener cerca de 50 años de edad y haber completado ocho años de educación."

Según el Consorcio Nacional de Soyeros ecuatoriano (ecuatoriano, 2014), la superficie sembrada de soya ha disminuido en los últimos 14 años. Según la Encuesta de Superficie, y Producción del 2014 realizado por el Instituto Nacional de Estadísticas y Censo (Encuesta de Superficie, 2014) se registraron 28,291 hectáreas sembradas de soya. Esto, a pesar de que la demanda nacional por el producto es importante, especialmente en la industria avícola. En este panorama, las importaciones de soya, especialmente en forma de torta, han constituido un rubro 
importante para el abastecimiento de la demanda nacional. El aumento de estas importaciones, en términos de volumen en los últimos 14 años haya sido importante; registrando una tasa de crecimiento entre los años 2000 y 2014 de $283 \%$.

Según el Reporte de Coyuntura 2015 del Banco Central del Ecuador (BCE, 2015) la última cosecha de soya verano 2014 mostró resultados negativos, repitiéndose por cuarto período seguido. La cantidad de superficie sembrada y su producción decayeron en $-25 \%$ y $-18 \%$, respectivamente. El estudio se basó a la provincia de Los Ríos y cantones próximos (sector de mayor producción de soya). De aquí deviene el grueso del cultivo; en orden de prelación Babahoyo, Montalvo y Quevedo. El cultivo de soya a nivel nacional es de 37 mil hectáreas, el 47.14\% menos que las 70,000 hectáreas que cosechaba en extensión Ecuador en años anteriores.

El presidente de la Corporación de Soyeros de Ecuador, indica que las cosechas han bajado su resultado, actualmente de cada hectárea se extrae de una tonelada a tonelada y media, tiempo atrás el rendimiento llegaba a dos toneladas y media inclusive a tres. Lo que se explica porque no se importan semillas de variedades de soya denominadas de alto rendimiento. También ha influido la corriente del Niño que aporta condiciones oscilantes.

\section{Conclusiones}

El cultivo de soya en el Ecuador es un cultivo transitorio, utilizado por los agricultores como opción de siembra de los cultivos de arroz y maíz duro seco durante la época de verano como lo consideran una opción para llenar un vacío en el ciclo de producción cuyo mayor interés gira entorno a otras especies, no existe un elevado grado de especialidad en su cultivo y no se consolida de una generación a otra lo que contrasta en relación a la demanda externa a nivel mundial, por lo que bien podría ser un nuevo eje en la producción y exportación del Ecuador. Queda abierto la incógnita de como proponer mayores incentivos para su cultivo por parte del Estado considerando su importancia y demostrada basta aplicación en diversas industrias. Articulando estrategias de competitividad para el agricultor a través del Ministerio de Agricultura Ganadería y Pesca MAGAP.

Las falencias que hallaron los productores de soya son de financiación, de asistencia técnica y altas tasas de tipo de interés, en semillas de variedad que presente mayor resistencia al clima y rendimiento. Eso en contraste con el comportamiento del precio de la soya a la baja, la falta de adecuados híbridos. El Banco Central del Ecuador / Publicaciones Técnicas refiere que el gobierno puede brindar apoyo al potencial del sector productor de soya al promover respetar el precio oficial y considerar estrategias para incentivar al sector industrial a absorber la producción nacional, en especial la proveniente de los pequeños agricultores.

A su vez las empresas privadas deben realizar estudios sobre las actividades complementarias para lograr ingresar a nuevos mercados y conseguir nuevos proyectos, tener una base de datos de la demanda en el exterior con los que se pueda contar para futuros proyectos y capacitar constantemente al personal técnico de acuerdo con las normas que exige el mercado internacional. 
Planificar y proyectar adecuadamente el tiempo que se dedicara a impulsar la producción de soya con variedades resistentes con el fin de cumplir con las expectativas de los compradores del exterior, estar constantemente en búsqueda de nuevos socios que puedan aportar con conocimientos y capitales.

Aprovechar la oportunidad que tiene Ecuador para generar mayor producción de soya y en acciones conjuntas entre el Estado y la empresa privada crear la industria que industrialice su procesamiento en productos derivados, siendo tan rica la amplia gama desde los básicos en la nutrición leche de soya, carne de soya, queso de soya, pasando por salsas, condimentos, galletas, confites, complementos nutricionales, incluso la industria farmacéutica nacional puede crecer en torno a la producción de soya. Y también se incluye la fuente de proteínas para la alimentación del ganado.

Actualmente toda esta demanda interna está siendo suplida con la importación de estos productos derivados de soya. Si se considera este gran potencial de la soya en primera instancia en la demanda interna. Y en una segunda etapa en producir para exportar considerando la gran demanda a nivel mundial que existe de los la soya y sus derivados procesados de calidad como se ha explorado en esta investigación.

\section{Bibliografía}

Agrodigital. (2015). Obtenido de http://www.agrodigital.com/

BCE, B. C. (2015). Reporte de Coyontura.

Bernal, Cesar A. (2010). Metodologia de la Investigacion. En C. A. Bernal, Metodologia de la investigacion (pág. 111). Bogota: Orlando Fernandez.

Bernal, Cesar A. (2010). Metodologia de la Investigacion. En B. Cesar, Mettodologia de la Investigacion. Bogota: Orlando Fernandez.

Cultivos, P. p. (2015). FAO. Obtenido de http://www.fao.org/docrep/004/y3557s/y3557s08.htm ecuatoriano, C. N. (2014). Informe.

Encuesta de Superficie, y. P. (2014). Instituto Nacional de Estadistica y Censos .

FAO. (2015). Perspectivas por sectores principales. Producción de cultivos. Obtenido de http://www.fao.org/worldfoodsituation/csdb/es/

Foodways, F. (2015). Consumo Humano.

Malthus, T. (1766-1834).

Ministerio de Agricultura, G. A. (2015). informe de Rendimientos Y Características De Soya En El Ecuador Verano. Guayaquil. 
ONU, O. d. (2015). Informe de perspectivas de la población mundial.

Soja Producción Mundial. (septiembre 2016 de 2016). https://www.produccionmundialsoja.com/, online. (S. P. Mundial, Editor, P. J. 2016, Productor, \& Soja Producción Mundial ) Recuperado el 3 de agosto de 2016, de https://www.produccionmundialsoja.com/: https://www.produccionmundialsoja.com/

Union Vegetariana Internacional. (2016). Obtenido de http://www.ivu.org/congress/wvc08/: http://www.ivu.org/congress/wvc08/ 